\title{
ON ALGEBRAS WITH CONVOLUTION STRUCTURES FOR LAGUERRE POLYNOMIALS
}

\author{
YÛICHI KANJIN
}

\begin{abstract}
In this paper we treat the convolution algebra connected with Laguerre polynomials which was constructed by Askey and Gasper [1]. For this algebra, we study the maximal ideal space, Wiener's general Tauberian theorem, spectral synthesis and Helson sets. We also study Sidon sets and idempotent measures for the algebras with dual convolution structures.
\end{abstract}

1. Introduction and preliminary results. Let $\alpha>-1$ and let $n$ be a nonnegative integer. Let $L_{n}^{\alpha}(x)$ denote the Laguerre polynomial defined by

$$
L_{n}^{\alpha}(x)=\frac{e^{x} x^{-\alpha}}{n !}\left(\frac{d}{d x}\right)^{n}\left(e^{-x} x^{n+\alpha}\right) .
$$

Laguerre polynomials have the following properties:

$$
\begin{gathered}
L_{n}^{\alpha}(0)=\left(\begin{array}{c}
n+\alpha \\
n
\end{array}\right) \\
\int_{0}^{\infty} L_{m}^{\alpha}(x) L_{n}^{\alpha}(x) e^{-x} x^{\alpha} d x=\Gamma(\alpha+1)\left(\begin{array}{c}
n+\alpha \\
n
\end{array}\right) \delta_{m n}
\end{gathered}
$$

where $\left(\begin{array}{c}p \\ q\end{array}\right)=p(p-1) \cdots(p-q+1) / q$ ! and $\delta_{m n}$ is Kronecker's symbol. Denote by $R_{n}^{\alpha}(x)$ the normalized Laguerre polynomial so that

$$
R_{n}^{\alpha}(x)=L_{n}^{\alpha}(x) / L_{n}^{\alpha}(0) .
$$

The purpose of this paper is to study some structures of convolution algebras connected with Laguerre polynomials, e.g., maximal ideal spaces, Helson sets, idempotent measures, spectral synthesis of the set of one point, etc.

Askey and Gaspar [1] proved the following:

(A) [1, Theorem 2] If $\alpha \geq-1 / 2$ and $\tau \geq 2$ or if $\alpha \geq \alpha_{0}=\left(-5+(17)^{1 / 2}\right) / 2$ and $\tau \geq 1$, then

$$
e^{-\tau x} R_{m}^{\alpha}(x) R_{n}^{\alpha}(x)=\sum_{k=0}^{\infty} D_{k}^{\alpha}(m, n ; \tau) R_{k}^{\alpha}(x), \quad x \geq 0
$$

with $D_{k}^{\alpha}(m, n ; \tau) \geq 0$, where the series $\sum_{k=0}^{\infty} D_{k}^{\alpha}(m, n ; \tau) R_{k}^{\alpha}(x)$ converges for every $x \geq 0$.

Received by the editors July 17,1985 .

1980 Mathematics Subject Classification. Primary 43A20, 42C10; Secondary 43A10, 43 A46.

Key words and phrases. Convolution structures, Laguerre polynomials, maximal ideal spaces, Helson sets, Sidon sets, spectral synthesis, idempotent measures.

(C)1986 American Mathematical Society $0002-9947 / 86 \$ 1.00+\$ .25$ per page 
They also constructed a convolution algebra as follows. Let $l$ be the space of absolutely convergent sequences $a=\left\{a_{n}\right\}_{n=0}^{\infty}, \sum_{n=0}^{\infty}\left|a_{n}\right|<\infty$ with norm $\|a\|=$ $\sum_{n=0}^{\infty}\left|a_{n}\right|$. For $a$ and $b$ in $l$, define the convolution $a * b$ by

$$
(a * b)_{k}=\sum_{m, n=0}^{\infty} a_{m} b_{n} D_{k}^{\alpha}(m, n ; \tau), \quad k=0,1,2, \ldots
$$

Then $\|a * b\| \leq\|a\|\|b\|$, since $\sum_{k=0}^{\infty}\left|D_{k}(m, n ; \tau)\right|=1$ by (A). Denote by $l^{(\alpha, \tau)}$ the algebra $l$ with the convolution $*$. Then $l^{(\alpha, \tau)}$ is a commutative Banach algebra.

We define the function $\hat{a}(x)$ on $[0, \infty)$ by

$$
\hat{a}(x)=\sum_{n=0}^{\infty} a_{n} R_{n}^{\alpha}(x) e^{-\tau x}
$$

for every $a=\left\{a_{n}\right\}_{n=0}^{\infty}$ in $l^{(\alpha, \tau)}$. Since $\left|R_{n}^{\alpha}(x) e^{-x}\right| \leq 1, x \geq 0$ for $\alpha \geq 1 / 2[1,(5.9)]$, the function $\hat{a}(x)$ is continuous on $[0, \infty)$ and $\lim _{x \rightarrow \infty} \hat{a}(x)=0$ for every $a$ in $l^{(\alpha, \tau)}$. We put

$$
A^{(\alpha, \tau)}=\left\{\hat{a} ; a \text { in } l^{(\alpha, \tau)}\right\} .
$$

In $\S 2$, first we will shw that $A^{(\alpha, \tau)}$ is a Banach algebra which is isomorphic and isometric to $l^{(\alpha, \tau)}$ if we introduce the product of pointwise multiplication of functions and the norm $\|\hat{a}\|=\|a\|$ to $A^{(\alpha, \tau)}$. Next we will determine the maximal ideal space of $A^{(\alpha, \tau)}$ and have a result analogous to Wiener's general Tauberian theorem. In $\S 3$, we will study problems of Helson sets and spectral synthesis for $A^{(\alpha, \tau)}$ which have originally arisen from the algebra $A(\mathbf{T})$ of absolutely convergent Fourier series (cf. Kahane [8]). By means of our results in $§ 3$, we may obtain that the structure of $A^{(\alpha, \tau)}$ is simpler than that of $A(\mathrm{~T})$ and is similar to that of the algebra of absolutely convergent Jacobi polynomial series or the algebra of Hankel transforms. See Igari and Uno [7] and Schwartz [11].

We will also consider the algebras with dual convolution structures for Laguerre polynomials.

Görlich and Markett [5] introduced the spaces $L_{W(\alpha)}^{p}, \alpha \geq 0$, of measurable functions on $[0, \infty)$ which are suitable for defining convolution structures for Laguerre polynomials $L_{n}^{\alpha}(x), \alpha \geq 0$. We will deal with the space $L_{W(\alpha)}^{1}$ and denote it briefly by $L_{\alpha}$;

$$
L_{\alpha}=\left\{f ;\|f\|=\int_{0}^{\infty}|f(x)| e^{-x / 2} x^{\alpha} d x<\infty\right\}, \quad \alpha \geq 0 .
$$

For $f$ and $g$ in $L_{\alpha}$, the convolution is defined by

$$
f * g(t)=\int_{0}^{\infty} T_{t}^{\alpha}(f ; x) g(x) e^{-x} x^{\alpha} d x, \quad t \geq 0
$$

where $T_{t}^{\alpha}(f ; x)$ is the Laguerre translation of $f$ given by

$$
\begin{aligned}
T_{t}^{\alpha}(f ; x)=\frac{\Gamma(\alpha+1) 2^{\alpha}}{(2 \pi)^{1 / 2}} \int_{0}^{\pi} f\left(x+t+2(x t)^{1 / 2} \cos \theta\right) \exp \left(-(x t)^{1 / 2} \cos \theta\right) \\
\\
\cdot \frac{J_{\alpha-1 / 2}\left((x t)^{1 / 2} \sin \theta\right)}{\left((x t)^{1 / 2} \sin \theta\right)^{\alpha-1 / 2}} \sin ^{2 \alpha} \theta d \theta
\end{aligned}
$$

for $x, t>0, T_{t}^{\alpha}(f ; 0)=f(t)$ for $t>0, T_{0}^{\alpha}(f ; x)=f(x)$ for $x \geq 0$. 
(B) [5, Theorem 1(ii)] Let $\alpha \geq 0$. Then $L_{\alpha}$ is a commutative Banach algebra; that is, $\|f * g\| \leq\|f\|\|g\|$ for $f$ and $g$ in $L_{\alpha}$.

Let $M[0, \infty)$ be the space of all bounded regular Borel measures on $[0, \infty)$. Then the space $M[0, \infty)$ is a Banach space with total variation norm $\|\mu\|=\int_{0}^{\infty} d|\mu|(x)$ for $\mu$ in $M[0, \infty)$.

In $\S 4$, we will introduce a convolution structure to $M[0, \infty)$ so that $L_{\alpha}$ is included in $M[0, \infty)$ as a closed ideal by the mapping $f \mapsto \mu_{f}$ of $L_{\alpha}$ into $M[0, \infty)$, where $d \mu_{f}(x)=f(x) e^{-x / 2} x^{\alpha} d x$, and denote by $M_{\alpha}$ the algebra $M[0, \infty)$ with this convolution structure. We will determine the maximal ideal spaces of $L_{\alpha}$ and $M_{\alpha}$. In $\S 5$, we will study idempotent measures in $M_{\alpha}$ and Sidon sets for $L_{\alpha}$. Although the Laguerre translation is not positive, our results show that the structures of $M_{\alpha}$ and $L_{\alpha}$ are similar to those of the algebras for ultraspherical polynomials or Jacobi polynomials with positive convolution structures. See Dunkl [2], Gasper [4], Igari $[\mathbf{6}]$, and also [11].

2. Algebras $l^{(\alpha, \tau)}$ and $A^{(\alpha, \tau)}$. Let $C_{c}^{\infty}$ be the space of infinitely differentiable functions with compact support in $[0, \infty)$. First, we prove two lemmas.

LEMMA 1. Let $\alpha>-1$ and let $p$ be a positive integer. Then there is a constant $C$ depending only on $\alpha$ and $p$ such that

$$
\left|\int_{0}^{\infty} f(x) L_{n}^{\alpha}(x) e^{-x} x^{\alpha} d x\right| \leq C n^{(\alpha-p) / 2} \sup _{0 \leq x}\left|\left(\frac{d}{d x}\right)^{p} f(x)\right|
$$

for $f \in C_{c}^{\infty}$ and $n \geq p$.

Proof. We use the identity

$$
n !\left(\frac{d}{d x}\right)^{m}\left(L_{n}^{\beta}(x) e^{-x} x^{\beta}\right)=(m+n) ! L_{m+n}^{\beta-m}(x) e^{-x} x^{\beta-m}
$$

(Erdélyi et al. [3, 10.12(28)]) and the inequality

$$
\begin{aligned}
\left|L_{n}^{\alpha}(x)\right| e^{-x / 2} x^{\alpha / 2} & \leq K n^{\alpha} x^{\alpha / 2} \quad(\text { for } 0 \leq x<1 / n) \\
& \leq K n^{\alpha / 2} x^{-1 / 4}\left(|\nu-x|+\nu^{1 / 3}\right)^{-1 / 4} \\
& \leq K n^{\alpha / 2} e^{-L x} \quad(\text { for } 2 \nu \leq x),
\end{aligned}
$$

where $\nu=4 n+2 \alpha+2$, and $K$ and $L$ are positive constants not depending on $n$ and $x$ (cf. Muckenhoupt $[9,(2.13)]$ ). Put $A=\int_{0}^{\infty} f(x) L_{n}^{\alpha}(x) e^{-x} x^{\alpha} d x$. Then by (4) and integration by parts, we have

$$
\begin{aligned}
A & =\frac{(n-p) !}{n !} \int_{0}^{\infty} f(x)\left(\frac{d}{d x}\right)^{p}\left(L_{n-p}^{\alpha+p}(x) e^{-x} x^{\alpha+p}\right) d x \\
& =\frac{(n-p) !(-1)^{p}}{n !} \int_{0}^{\infty}\left\{\left(\frac{d}{d x}\right)^{p} f(x)\right\} L_{n-p}^{\alpha+p}(x) e^{-x} x^{\alpha+p} d x
\end{aligned}
$$

since $f$ has a compact support and

$$
\left.\left(\frac{d}{d x}\right)^{p-j}\left(L_{n-p}^{\alpha+p}(x) e^{-x} x^{\alpha+p}\right)\right|_{x=0}=0
$$


for $j=1,2,3, \ldots, p$. Thus

$$
|A| \leq \frac{(n-p) !}{n !} \cdot \int_{0}^{\infty}\left|L_{n-p}^{\alpha+p}(x)\right| e^{-x} x^{\alpha+p} d x \cdot \sup _{0 \leq x}\left|\left(\frac{d}{d x}\right)^{p} f(x)\right|
$$

We write

$$
\begin{aligned}
\int_{0}^{\infty} & \left|L_{n-p}^{\alpha+p}(x)\right| e^{-x} x^{\alpha+p} d x \\
& =\left(\int_{0}^{1 /(n-p)}+\int_{1 /(n-p)}^{2 \nu_{p}}+\int_{2 \nu_{p}}^{\infty}\right)\left|L_{n-p}^{\alpha+p}(x)\right| e^{-x} x^{\alpha+p} d x \\
& =I_{1}+I_{2}+I_{3},
\end{aligned}
$$

where $\nu_{p}=4(n-p)+2(\alpha+p)+2$. By (5), we have

$$
\begin{aligned}
& I_{1} \leq K(n-p)^{\alpha+p} \int_{0}^{1 /(n-p)} e^{-x / 2} x^{\alpha+p} d x \\
& I_{2} \leq K(n-p)^{(\alpha+p) / 2} \int_{0}^{\infty} e^{-x / 2} x^{(\alpha+p-1 / 2) / 2} d x \\
& I_{3} \leq K(n-p)^{(\alpha+p) / 2} \int_{0}^{\infty} e^{-(L+1 / 2) x} x^{(\alpha+p) / 2} d x
\end{aligned}
$$

and these inequalities complete the proof.

LEMMA 2. Let $\alpha \geq-1 / 2$ and let $\tau$ be a real number.

(i) Let $f$ be in $C_{c}^{\infty}$ and put

$$
a_{n}=\frac{1}{\Gamma(\alpha+1)} \int_{0}^{\infty} f(x) e^{\tau x} L_{n}^{\alpha}(x) e^{-x} x^{\alpha} d x
$$

Then the sequence $\left\{a_{n}\right\}_{n=0}^{\infty}$ belongs to $l$, and

$$
f(x)=\sum_{n=0}^{\infty} a_{n} R_{n}^{\alpha}(x) e^{-\tau x}
$$

for every $x \geq 0$.

(ii) The sequences $\left\{a_{n}\right\}_{n=0}^{\infty}$ of all $f$ in $C_{c}^{\infty}$ are dense in l.

ProOF. By Lemma 1, we have

$$
\left|a_{n}\right| \leq \Gamma(\alpha+1)^{-1} C n^{(\alpha-p)-2} \sup _{0 \leq x}\left|\left(\frac{d}{d x}\right)^{p}\left(f(x) e^{\tau x}\right)\right| .
$$

Since $p$ is arbitrary, we have $\sum_{n=0}^{\infty}\left|a_{n}\right|<\infty$. By the equiconvergence theorem for Laguerre series for $x>0$ and the summability theorem for Laguerre series at $x=0$ (cf. Szegö [12, Theorems 9.1.5 and 9.1.7]), we have $f(x)=\sum_{n=0}^{\infty} a_{n} R_{n}^{\alpha}(x) e^{-\tau x}$ for every $x \geq 0$.

To prove (ii), it is enough to show that, for every $j=0,1,2, \ldots, c(j)$ is approximated by sequences $\left\{a_{n}\right\}_{n=0}^{\infty}$ of functions in $C_{c}^{\infty}$, where $c(j)_{n}=0$ for $n \neq j$ and $c(j)_{n}=1$ for $n=j$. Let $h(x)$ be a function of $C_{c}^{\infty}$ such that $h(x)=1$ for $0 \leq x \leq 1,0<h(x)<1$ for $1<x<2$ and $h(x)=0$ for $2 \leq x$. Put 
$f_{k}(x)=h(x / k) R_{j}^{\alpha}(x) e^{-\tau x}$ for every $k=1,2,3, \ldots$ Then $f_{k}$ belongs to $C_{c}^{\infty}$. Define the sequence $a^{(k)}=\left\{a_{n}^{(k)}\right\}_{n=0}^{\infty}$ by

$$
a_{n}^{(k)}=\frac{1}{\Gamma(\alpha+1)} \int_{0}^{\infty} f_{k}(x) e^{\tau x} L_{n}^{\alpha}(x) e^{-x} x^{\alpha} d x .
$$

We will show that $a^{(k)}$ converges to $c(j)$ in $l$ as $k$ tends to infinity. By Lemma 1 , we have

$$
\begin{aligned}
\left|a_{n}^{(k)}-c(j)_{n}\right| & =\frac{1}{\Gamma(\alpha+1)}\left|\int_{0}^{\infty}\left(h\left(\frac{x}{k}\right)-1\right) R_{j}^{\alpha}(x) L_{n}^{\alpha}(x) e^{-x} x^{\alpha} d x\right| \\
& \leq \frac{1}{\Gamma(\alpha+1)} C n^{(\alpha-p) / 2} \sup _{0 \leq x}\left|\left(\frac{d}{d x}\right)^{p}\left(h\left(\frac{x}{k}\right)-1\right) R_{j}^{\alpha}(x)\right|
\end{aligned}
$$

for $n \geq p$, and thus $\left|a_{n}^{(k)}-c(j)_{n}\right| \leq C^{\prime} n^{(\alpha-p) / 2}$ for $n \geq p$ with a constant $C^{\prime}$ not depending on $n$ and $k$. Since $a_{n}^{(k)}-c(j)_{n}$ converges to 0 as $k$ tends to infinity and the series $\sum n^{(\alpha-p) / 2}$ converges for large $p$, we have

$$
\lim _{k \rightarrow \infty} \sum_{n=0}^{\infty}\left|a_{n}^{(k)}-c(j)_{n}\right|=\sum_{n=0}^{\infty} \lim _{k \rightarrow \infty}\left|a_{n}^{(k)}-c(j)_{n}\right|=0 .
$$

This completes the proof.

Let $\alpha \geq-1 / 2$ and $\tau \geq 2$ or let $\alpha \geq \alpha_{0}=\left(-5+(17)^{1 / 2}\right) / 2$ and $\tau \geq 1$. By (1) and (2), we have

$$
\begin{aligned}
\hat{a}(x) \hat{b}(x) & =\sum_{m, n} a_{m} b_{n} R_{m}^{\alpha}(x) R_{n}^{\alpha}(x) e^{-2 \tau x} \\
& =\sum_{k}\left\{\sum_{m, n} a_{m} b_{n} D_{k}^{\alpha}(m, n ; \tau)\right\} R_{k}^{\alpha}(x) e^{-\tau x} \\
& =\sum_{k}(a * b)_{k} R_{k}^{\alpha}(x) e^{-\tau x}=(a * b)^{-}(x)
\end{aligned}
$$

for every $x \geq 0$. This shows that $A^{(\alpha, \tau)}$ is an algebra with the product of pointwise multiplication of functions. Let $a$ be in $l^{(\alpha, \tau)}$ and suppose $\hat{a}(x)=0$ on $[0, \infty)$. Then, for all $f$ in $C_{c}^{\infty}$, we have

$$
\begin{aligned}
0 & =\int_{0}^{\infty} f(x) \hat{a}(x) e^{\tau x} e^{-x} x^{\alpha} d x \\
& =\Gamma(\alpha+1) \sum_{n=0}^{\infty}\left(\begin{array}{c}
n+\alpha \\
n
\end{array}\right)^{-1} a_{n} b_{n}
\end{aligned}
$$

where

$$
b_{n}=\frac{1}{\Gamma(\alpha+1)} \int_{0}^{\infty} f(x) L_{n}^{\alpha}(x) e^{-x} x^{\alpha} d x .
$$

Since the sequence $\left\{b_{n}\right\}_{n=0}^{\infty}$ is dense in $l$ by Lemma 2(ii), we have $a_{n}=0$ for all $n$. This enables us to define the norm of $\hat{a}$ in $A^{(\alpha, \tau)}$ by $\|\hat{a}\|=\|a\|$. Then we have the following. 
Proposition 1. Let $\alpha \geq-1 / 2$ and $\tau \geq 2$, or let $\alpha \geq \alpha_{0}=\left(-5+(17)^{1 / 2}\right) / 2$ and $\tau \geq 1$. Then $A^{(\alpha, \tau)}$ is the commutative semisimple Banach algebra with no unit

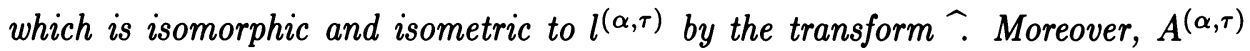
consists of continuous functions on $[0, \infty)$ vanishing at infinity, and includes $C_{c}^{\infty}$ as a dense subset. by

Let $x$ be in $[0, \infty)$ and define the mapping $\chi_{x}$ of $A^{(\alpha, \tau)}$ into the complex numbers

$$
\chi_{x}: f \mapsto f(x), \quad f \text { in } A^{(\alpha, \tau)} .
$$

Then $\chi_{x}$ is a multiplicative linear functional on $A^{(\alpha, \tau)}$. We denote by $\mathcal{M}\left(A^{(\alpha, \tau)}\right)$ the maximal ideal space of $A^{(\alpha, \tau)}$ and define the mapping $\iota$ of $[0, \infty)$ into $\mathcal{M}\left(A^{(\alpha, \tau)}\right)$ by $\iota: x \rightarrow \chi_{x}$.

THEOREM 1. Let $\alpha \geq-1 / 2$ and $\tau \geq 2$ or let $\alpha \geq \alpha_{0}$ and $\tau \geq 1$. Then the maximal ideal space $\mathcal{M}\left(\bar{A}^{(\alpha, \tau)}\right)$ is homeomorphic to the interval $[0, \infty)$, and the Gelfand transform of $f$ in $A^{(\alpha, \tau)}$ is given by $f$ itself.

Proof. Clearly, $\chi_{x} \neq \chi_{y}$ if $x \neq y$. Since both spaces $[0, \infty)$ and $\mathcal{M}\left(A^{(\alpha, \tau)}\right)$ are locally compact Hausdorff spaces, it is enough to show that the mapping $\iota$ is surjective. Let $\chi$ be a multiplicative linear functional on $A^{(\alpha, \tau)}$. Suppose that $f$ belongs to $A^{(\alpha, \tau)}$. Then

$$
f(x)=\sum_{n=0}^{\infty} a_{n} R_{n}^{\alpha}(x) e^{-\tau x}, \quad \sum_{n=0}^{\infty}\left|a_{n}\right|<\infty .
$$

If we define $c(n), n=0,1,2, \ldots$, by $c(n)_{k}=0$ for $k \neq n$ and $c(n)_{k}=1$ for $k=n$, then we have

$$
c(n)^{\Upsilon}(x)=R_{n}^{\alpha}(x) e^{-\tau x}, \quad\|c(n)\|=1 \text { for all } n .
$$

To complete the proof, it is enough to show that

$$
\chi\left(c(n)^{\uparrow}\right)=R_{n}^{\alpha}\left(x_{0}\right) e^{-\tau x_{0}}, \quad n=0,1,2, \ldots,
$$

for some $x_{0}$ in $[0, \infty)$.

By the recurrence formula

$$
(n+\alpha) R_{n}^{\alpha}(x)=(-x+2 n+\alpha-1) R_{n-1}^{\alpha}(x)-(n-1) R_{n-2}^{\alpha}(x),
$$

$n=2,3,4, \ldots, R_{0}^{\alpha}(x)=1$ and $R_{1}^{\alpha}(x)=1-x /(\alpha+1)[12,(5.1 .10)]$, we have

$$
\begin{aligned}
(\alpha+1) c(1)^{\curlyvee}(x) c(n-1)^{\curlyvee}(x)= & (n-1) c(n-2)^{\uparrow}(x) c(0)^{\curlyvee}(x) \\
& -2(n-1) c(n-1)^{\curlyvee}(x) c(0)^{\curlyvee}(x) \\
& +(n+\alpha) c(n)^{\curlyvee}(x) c(0)^{\curlyvee}(x), \quad x \geq 0,
\end{aligned}
$$

and thus

$$
\text { (8) } \begin{aligned}
(\alpha+1) \chi\left(c(1)^{\uparrow}\right) \chi\left(c(n-1)^{\uparrow}\right)=(n-1) \chi\left(c(n-2)^{\uparrow}\right) \chi\left(c(0)^{\uparrow}\right) \\
-2(n-1) \chi\left(c(n-1)^{\uparrow}\right) \chi\left(c(0)^{\uparrow}\right)+(n+\alpha) \chi\left(c(n)^{\uparrow}\right) \chi\left(c(0)^{\uparrow}\right) .
\end{aligned}
$$

Now we claim that $\chi\left(c(0)^{\uparrow}\right) \neq 0$. For, since

$$
\chi(f)=\chi((f / c(0)\urcorner c(0)\urcorner=\chi(f / c(0)\urcorner \chi(c(0)\urcorner
$$


for all $f$ in $C_{c}^{\infty}$ which is dense in $A^{(\alpha, \tau)}$, we have that $\chi$ is trivial if $\chi\left(c(0)^{-}\right)=0$. Put $g_{n}=\chi\left(c(0)^{\wedge}\right)^{-1} c(n)^{\wedge}, n=0,1,2, \ldots$, and choose the unique complex number $x_{0}$ so that $\chi\left(g_{1}\right)=1-x_{0} /(\alpha+1)$. Then, by (8), we have

$$
(n+\alpha) \chi\left(g_{n}\right)=\left(-x_{0}+2 n+\alpha-1\right) \chi\left(g_{n-1}\right)-(n-1) \chi\left(g_{n-2}\right),
$$

$n=2,3,4, \ldots, \chi\left(g_{0}\right)=1$ and $\chi\left(g_{1}\right)=1-x_{0} /(\alpha+1)$. This shows that the sequence $\chi\left(g_{n}\right), n=0,1,2, \ldots$, satisfies the recurrence formula (7) with $x=x_{0}$. Thus we have

$$
\chi\left(g_{n}\right)=R_{n}^{\alpha}\left(x_{0}\right), \quad n=0,1,2, \ldots
$$

Since the norm of a multiplicative linear functional is at most one, it follows that $\left|R_{n}^{\alpha}\left(x_{0}\right)\right| \leq 1$ for all $n$ by (6). If $x_{0}$ does not belong to $[0, \infty)$, then $\lim _{n \rightarrow \infty}\left|R_{n}^{\alpha}\left(x_{0}\right)\right|$ $=\infty$ by Perron's formula in the complex domain [12, Theorem 8.22.3]. This shows that $x_{0}$ belongs to $[0, \infty)$. By (1) and (9), we have

$$
\begin{aligned}
\chi\left(c(0)^{-}\right) & =\sum_{k=0}^{\infty} D_{k}^{\alpha}(0,0 ; \tau) \chi\left(g_{k}\right) \\
& =\sum_{k=0}^{\infty} D_{k}^{\alpha}(0,0 ; \tau) R_{n}^{\alpha}\left(x_{0}\right)=e^{-\tau x_{0}}
\end{aligned}
$$

and therefore

$$
\chi\left(c(n)^{\top}\right)=R_{n}^{\alpha}\left(x_{0}\right) e^{-\tau x_{0}} .
$$

This completes the proof.

By the theorem and Lemma 2(ii), we have the following.

COROLlaRY 1. The semisimple Banach algebra $A^{(\alpha, \tau)}$ is regular.

Let $E$ be a compact subset of $[0, \infty)$ and define $I(E)=\left\{f \in A^{(\alpha, \tau)} ; f=0\right.$ on $\left.E\right\}$. Then $I(E)$ is a closed ideal in $A^{(\alpha, \tau)}$. The application of the usual Banach algebra proof of the Wiener-Lévy theorem to the quotient algebra $A^{(\alpha, \tau)} / I(E)$ yields the following.

COROLLARY 2. Let $f$ be in $A^{(\alpha, \tau)}$. Suppose that $f \neq 0$ on a compact subset $E$ of $[0, \infty)$. Then there is a function $g$ in $A^{(\alpha, \tau)}$ such that $f(x) g(x)=1$ for $x$ in $E$.

This corollary and Lemma 2(ii) yield the following result, analogous to Wiener's general Tauberian theorem (cf. Rudin $[\mathbf{1 0}, 7.2])$.

COROLlaRY 3. Let $f$ be in $A^{(\alpha, \tau)}$ and suppose that $f(x) \neq 0$ for all $x$ in $[0, \infty)$. Then $f$ is contained in no proper closed ideal in $A^{(\alpha, \tau)}$.

PrOOF. Let $h$ be in $C_{c}^{\infty}$ and let $E$ be the support of $h$. By Corollary 2, we have a function $g$ in $A^{(\alpha, \tau)}$ such that $f g=1$ on $E$. It follows from $h f g=h$ that an ideal containing $f$ includes $C_{c}^{\infty}$. By Lemma 2(ii), a closed ideal containing $f$ coincides with $A^{(\alpha, \tau)}$.

3. Spectral synthesis and Helson sets. Let $E$ be a closed subset of $[0, \infty)$, and let $I(E)$ be the closed ideal of $f$ in $A^{(\alpha, \tau)}$ such that $f=0$ on $E$. Denote by $J(E)$ the ideal of $f$ in $A^{(\alpha, \tau)}$ such that $f=0$ on a neighborhood of $E$. If $J(E)$ is dense in $I(E)$, then $E$ is called a set of spectral synthesis for $A^{(\alpha, \tau)}$. By an argument similar to that used for Schwartz's example in the Euclidean space of three dimension (cf. $[\mathbf{1 0}, 7.3]$ ), we have the following. 
THEOREM 2. Let $\tau \geq 1$. If $\alpha \geq 1 / 2$ and $x_{0}$ is in the open interval $(0, \infty)$, then the singleton $\left\{x_{0}\right\}$ is not a set of spectral synthesis for $A^{(\alpha, \tau)}$.

PROOF. Let $k$ be the greatest integer not exceeding $\alpha+1 / 2$. It follows from the identity $(d / d x) L_{n}^{\alpha}(x)=-L_{n-1}^{\alpha+1}(x)[12,(5.1 .14)]$ that there is a positive constant $C$ and a neighborhood $V$ of $x_{0}$ such that $\left|(d / d x)^{j}\left(R_{n}^{\alpha}(x) e^{-\tau x}\right)\right| \leq C$ on $V$ for $j=1,2,3, \ldots, k$ and all $n$. This implies that every $f$ in $A^{(\alpha, \tau)}$ is $k$-times continuously differentiable on $(0, \infty)$. Let $I_{1}=\left\{f \in A^{(\alpha, \tau)} ; f\left(x_{0}\right)=0\right\}$ and $I_{2}=\left\{f \in A^{(\alpha, \tau)} ; f\left(x_{0}\right)=(d f / d x)\left(x_{0}\right)=0\right\}$. Then $I_{1}$ and $I_{2}$ are distinct closed ideals for $\alpha \geq 1 / 2$. The proof is complete.

A closed subset $E$ of $[0, \infty)$ will be called a Helson set with respect to the algebra $A^{(\alpha, \tau)}$ if, for every continuous function $g$ on $E$ vanishing at infinity, there is a function $f$ in $A^{(\alpha, \tau)}$ such that $f=g$ on $E$.

The following theorem is suggested by the characterization of Helson sets with respect to the algebra of absolutely convergent Jacobi polynomial series $[\mathbf{7}$, Theorem 2].

THEOREM 3. Let $\alpha>-1 / 2$ and $\tau \geq 2$, or let $\alpha \geq \alpha_{0}$ and $\tau \geq 1$. Then every Helson set with respect to $A^{(\alpha, \tau)}$ is finite.

PROOF. Every finite set is a Helson set with respect to $A^{(\alpha, \tau)}$ since $A^{(\alpha, \tau)}$ is regular.

Conversely, let $E$ be a Helson set with respect to $A^{(\alpha, \tau)}$. Suppose that $E$ is infinite. Then there are a sequence $\left\{x_{j}\right\}_{j=1}^{\infty}$ in $E \cap(0, \infty)$, such that $x_{i} \neq x_{j}$ for $i \neq j$, and a point $x_{0}$ such that $\lim _{j \rightarrow \infty} x_{j}=x_{0}$ and $0 \leq x_{0} \leq \infty$. Let $Q(E)$ be the quotient algebra $A^{(\alpha, \tau)} / I(E)$ with quotient norm $\|\cdot\|_{Q(E)}$, and let $C_{0}(E)$ be the Banach algebra of continuous functions on $E$ vanishing at infinity with uniform norm $\|\cdot\|_{C_{0}(E)}$. Since $E$ is a Helson set with respect to $A^{(\alpha, \tau)}$, it follows that $Q(E)$ is isomorphic to $C_{0}(E)$ and the norms in $Q(E)$ and in $C_{0}(E)$ are equivalent. Let $g_{k}$ be a function in $C_{0}(E)$ such that $g_{k}\left(x_{2 j}\right)=1$ and $g_{k}\left(x_{2 j-1}\right)=0$ for $j=1,2,3, \ldots, k$, $g_{k}\left(x_{j}\right)=0$ for $j=2 k+1,2 k+2, \ldots$ and $\left\|g_{k}\right\|_{C_{0}(E)}=1$. By the norm equivalence and the definition of quotient norm, we can choose a function $f_{k}$ in $A^{(\alpha, \tau)}$ for every $k$ so that $f_{k}(x)=g_{k}(x)$ on $E$ and $\left\|f_{k}\right\| \leq C$ with a constant $C$ not depending on $k$. Since $A^{(\alpha, \tau)}$ can be regarded as the dual of the space $c_{0}$ of sequences vanishing at infinity, the sequence $\left\{f_{k}\right\}_{k=1}^{\infty}$ has a subsequence, say also $\left\{f_{k}\right\}_{k=1}^{\infty}$, converging to a function $f$ in $A^{(\alpha, \tau)}$ in the weak ${ }^{*}$ topology $\sigma\left(A^{(\alpha, \tau)}, c_{0}\right)$. If $\alpha>-1 / 2$, then $R_{n}^{\alpha}(x) \rightarrow 0$ as $n \rightarrow \infty$ for every $x$ in $(0, \infty)[12,(7.6 .8)]$. Thus we have $f_{k}(x) \rightarrow f(x)$ as $k \rightarrow \infty$ for every $x$ in $(0, \infty)$ by the definition of weak* topology $\sigma\left(A^{(\alpha, \tau)}, c_{0}\right)$, and in particular $f\left(x_{2 j}\right)=1$ and $f\left(x_{2 j-1}\right)=0$ for all $j$. This contradicts the continuity of $f$ on $[0, \infty)$ in the case $x_{0}$ in $[0, \infty)$ and contradicts the vanishing at infinity of $f$ in the case $x_{0}=\infty$. The proof is complete.

4. Algebras $L_{\alpha}$ and $M_{\alpha}$. Let $\alpha>-1 / 2$. If we put $z=x+t+2(x t)^{1 / 2} \cos \theta$, $0 \leq \theta \leq \pi$ in (3), then we have the following for $x, t>0$ (cf. [5]):

$$
\begin{aligned}
(x t)^{1 / 2} \sin \theta & =\left\{2(x t+x z+t z)-x^{2}-t^{2}-z^{2}\right\}^{1 / 2} / 2 \\
& =\rho(x, t, z), \quad \text { say. } \\
T_{t}^{\alpha}(f ; x) & =\int_{0}^{\infty} f(z) K(x, t, z) e^{-z} z^{\alpha} d z
\end{aligned}
$$




$$
K(x, t, z)=\left\{\begin{array}{l}
\frac{\Gamma(\alpha+1) 2^{\alpha-1}}{(2 \pi)^{1 / 2}(x t z)^{\alpha}} e^{(x+t+z) / 2} \cdot J_{\alpha-1 / 2}(\rho(x, t, z)) \rho(x, t, z)^{\alpha-1 / 2} \\
0,
\end{array}\right.
$$

where the first value is assigned only if $2(x t+t z+z x)-x^{2}-t^{2}-z^{2}>0$.

The kernel $K(x, t, z)$ satisfies the following.

(C) $[5$, Lemma 1(ii)] Let $\alpha \geq 0$. Then

$$
\int_{0}^{\infty}|K(x, t, z)| e^{-z / 2} z^{\alpha} d z \leq e^{(x+t) / 2}
$$

for $x, t>0$.

Let $M^{\prime}$ be the space $\{\mu \in M[0, \infty) ;|\mu|(\{0\})=0\}$, and let $\mu$ and $\nu$ be in $M^{\prime}$. We define the convolution $\mu * \nu$ for $\alpha \geq 0$ by

$$
\mu * \nu(E)=\int_{0}^{\infty} \int_{0}^{\infty}\left\{\int_{E} K(x, t, z) e^{-z / 2} z^{\alpha} d z\right\} e^{-(x+t) / 2} d \mu(x) d \nu(t)
$$

for every Borel set $E$ of $(0, \infty)$. Then $\mu * \nu$ belongs to $M^{\prime}$ and $\|\mu * \nu\| \leq\|\mu\|\|\nu\|$ by (C). We denote by $M_{\alpha}^{\prime}$ the algebra $M^{\prime}$ with this convolution. Every $\mu$ in $M[0, \infty)$ has the unique decomposition $\mu=\nu+\mu(\{0\}) \delta_{0}$, where $\nu$ is in $M_{\alpha}^{\prime}$ and $\delta_{0}$ is the measure with the unit mass at the point 0 . We extend the convolution to all of $M[0, \infty)$ by treating $\delta_{0}$ as a unit. This algebra is a commutative Banach algebra with a unit and is denoted by $M_{\alpha}$. We identify $L_{\alpha}$ with its image by the mapping $f \mapsto \mu_{f}, d \mu_{f}(x)=f(x) e^{-x / 2} x^{\alpha} d x$. Then $L_{\alpha}$ is a closed subalgebra of $M_{\alpha}^{\prime}$ since $\mu_{f} * \mu_{g}=f * g$ and $\left\|\mu_{f}\right\|=\|f\|$.

The Fourier Laguerre coefficient $\hat{f}(n)$ of $f$ in $L_{\alpha}$ is defined by

$$
\hat{f}(n)=\int_{0}^{\infty} f(x) R_{n}^{\alpha}(x) e^{-x} x^{\alpha} d x
$$

for every $n=0,1,2, \ldots$ We define the Fourier-Stieltjes Laguerre coefficient $\hat{\mu}(n)$ of $\mu$ in $M_{\alpha}$ by

$$
\hat{\mu}(n)=\int_{0}^{\infty} R_{n}^{\alpha}(x) e^{-x / 2} d \mu(x)
$$

for every $n$. Then we have $\hat{\mu}_{f}(n)=\hat{f}(n)$, and

$$
(f * g) \uparrow(n)=\hat{f}(n) \hat{g}(n), \quad(\mu * \nu) \uparrow(n)=\hat{\mu}(n) \hat{\nu}(n)
$$

for $f, g$ in $L_{\alpha}$ and $\mu, \nu$ in $M_{\alpha}$ by the identity

$$
T_{t}^{\alpha}\left(R_{n}^{\alpha} ; x\right)=R_{n}^{\alpha}(x) R_{n}^{\alpha}(t), \quad \alpha>-1 / 2 \quad \text { (Watson [13]). }
$$

LEMMA 3. Let $\alpha \geq 0$.

(i) $|\hat{\mu}(n)| \leq\|\mu\|$ for $\mu$ in $M_{\alpha}$.

(ii) If a measure $\mu$ in $M_{\alpha}$ satisfies that $\hat{\mu}(n)=0$ for all $n$, then $\mu=0$.

Proof. The inequality $\left|R_{n}^{\alpha}(x) e^{-x / 2}\right| \leq 1, \alpha \geq 0[3,10.18(14)]$ implies (i). To prove (ii), it is enough to show that $\int_{0}^{\infty} f(x) d \mu(x)=0$ for every $f$ in $C_{c}^{\infty}$. By Lemma 2(i), we have $f(x)=\sum_{n=0}^{\infty} a_{n} R_{n}^{\alpha}(x) e^{-x / 2}$ with $\sum_{n=0}^{\infty}\left|a_{n}\right|<\infty$. Thus we have $\int_{0}^{\infty} f(x) d \mu(x)=\sum_{n=0}^{\infty} a_{n} \hat{\mu}(n)=0$ by the Lebesgue convergence theorem since

$$
\sum_{n=0}^{\infty} \int_{0}^{\infty}\left|a_{n} R_{n}^{\alpha}(x) e^{-x / 2}\right| d|\mu|(x)=\sum_{n=0}^{\infty}\left|a_{n}\right|\|\mu\|<\infty .
$$


Now we consider the maximal ideal space of $L_{\alpha}$. For every $n=0,1,2, \ldots$, we define the functional $\chi_{n}$ by

$$
\chi_{n}(f)=\hat{f}(n), \quad f \text { in } L_{\alpha}
$$

Then $\chi_{n}$ is a multiplicative linear functional on $L_{\alpha}$ by (11).

PROPOSITION 2. Let $\alpha \geq 0$. Then the maximal ideal space of $L_{\alpha}$ is the space $\left\{\chi_{n} ; n=0,1,2, \ldots\right\}$ with the discrete topology.

PROOF. Let $\chi$ be a multiplicative linear functional on $L_{\alpha}$. We have an integer $n_{0} \geq 0$ such that $\chi\left(R_{n_{0}}^{\alpha}\right) \neq 0$. For, if $\chi\left(R_{n}^{\alpha}\right)=0$ for all $n$, then we have $\chi(f)=0$ for all $f$ in $L_{\alpha}$ since the Cesàro mean of order $\delta>\alpha+1 / 2$ of the Fourier Laguerre series converges in $L_{\alpha}$ by [5, (3.7)]. It follows from the orthogonality of Laguerre polynomials that $n_{0}$ is unique, and also $f * R_{n_{0}}^{\alpha}=\hat{f}\left(n_{0}\right) R_{n_{0}}^{\alpha}$ for $f$ in $L_{\alpha}$. This implies that $\chi(f) \chi\left(R_{n_{0}}^{\alpha}\right)=\hat{f}\left(n_{0}\right) \chi\left(R_{n_{0}}^{\alpha}\right)$, and thus $\chi(f)=\hat{f}\left(n_{0}\right)$. Since the Gelfand topology is clearly discrete, the proof is complete.

We denote by $\mathcal{M}\left(M_{\alpha}\right)$ and $\mathcal{M}\left(M_{\alpha}^{\prime}\right)$ the maximal ideal spaces of $M_{\alpha}$ and $M_{\alpha}^{\prime}$, respectively. The algebra $M_{\alpha}$ has the direct sum decomposition $M_{\alpha}=M_{\alpha}^{\prime} \oplus$ $C \delta_{0}$. Here, $C \delta_{0}$ is the closed subalgebra of $M_{\alpha}$ generated by $\delta_{0}$. It follows from the definition of the convolution that $M_{\alpha}^{\prime}$ is a closed ideal in $M_{\alpha}$. Thus $\mathcal{M}\left(M_{\alpha}\right)$ is the one point compactification $\left\{\chi_{\infty}\right\} \cup \mathcal{M}\left(M_{\alpha}^{\prime}\right)$ of $\mathcal{M}\left(M_{\alpha}^{\prime}\right)$, where $\chi_{\infty}$ is the multiplicative linear functional on $M_{\alpha}$ such that $\chi_{\infty}(\mu)=\mu(\{0\})$ for $\mu$ in $M_{\alpha}$. The next two lemmas are essential to the following discussion. See [2] and also [11].

LEMMA 4. Let $\alpha \geq 0$. If $\mu$ and $\nu$ are in $M_{\alpha}^{\prime}$, then $\mu * \nu$ is in $L_{\alpha}$.

ProOF. Let $E$ be a Borel set in $(0, \infty)$ such that $\int_{E} e^{-x / 2} x^{\alpha} d x=0$. For all $x, t>0$, the braces \{\} in the definition (10) of the convolution of $\mu$ and $\nu$ are zero since $K(x, t, z)$ are integrable with respect to the measure $e^{-z / 2} z^{\alpha} d z$ by (C). Thus we have $\mu * \nu(E)=0$; that is, $\mu * \nu$ is absolutely continuous with respect to $e^{-z / 2} z^{\alpha} d z$. This shows that $\mu * \nu$ in $L_{\alpha}$.

LEMMA 5. Let $\alpha \geq 0$. If $\mu$ is in $M_{\alpha}^{\prime}$, then $\lim _{n \rightarrow \infty} \hat{\mu}(n)=0$. In particular, $\chi_{\infty}(\mu)=\lim _{n \rightarrow \infty} \hat{\mu}(n)$ for $\mu$ in $M_{\alpha}$.

Proof. Since $\mu * \mu$ is in $L_{\alpha}$ for $\mu$ in $M_{\alpha}^{\prime}$ by Lemma 4 , it is enough to show that $\lim _{n \rightarrow \infty} \hat{f}(n)=0$ for every $f$ in $L_{\alpha}$. The usual argument in the Riemann-Lebesgue theorem implies that $\lim _{n \rightarrow \infty} \hat{f}(n)=0$ by Lemma $3(\mathrm{i})$ and the convergence of the Cesàro mean of order $\delta>\alpha+1 / 2$ in $L_{\alpha}$.

We can determine the maximal ideal spaces $\mathcal{M}\left(M_{\alpha}^{\prime}\right)$ and $\mathcal{M}\left(M_{\alpha}\right)$ by using Lemma 4.

THEOREM 4. Let $\alpha \geq 0$.

(i) The maximal ideal space $\mathcal{M}\left(M_{\alpha}^{\prime}\right)$ of $M_{\alpha}^{\prime}$ is the space $\left\{\chi_{n} ; n=0,1,2, \ldots\right\}$ with the discrete topology, where $\chi_{n}(\mu)=\hat{\mu}(n)$ for $\mu$ in $M_{\alpha}^{\prime}$.

(ii) The maximal ideal space of $\mathcal{M}\left(M_{\alpha}\right)$ of $M_{\alpha}$ is the space $\left\{\chi_{n} ; n=0,1,2, \ldots\right\} \cup$ $\left\{\chi_{\infty}\right\}$ with topology of the one point compactification of $\left\{\chi_{n} ; n=0,1,2, \ldots\right\}$.

PROOF. Every $\chi_{n}$ is a multiplicative linear functional on $M_{\alpha}^{\prime}$ by (11). Conversely, let $\chi$ be a multiplicative linear functional on $M_{\alpha}^{\prime}$. Let $\nu$ be a measure of $M_{\alpha}^{\prime}$ such that $\chi(\nu) \neq 0$. Since $\nu * \nu$ is in $L_{\alpha}$ by Lemma 4 , the restriction of $\chi$ to $L_{\alpha}$ 
is nontrivial. Thus there is a unique element $\chi_{n}$ of $\mathcal{M}\left(L_{\alpha}\right)$ such that $\chi(f)=\chi_{n}(f)$ for all $f$ in $L_{\alpha}$. Since $\mu * \nu$ in $L_{\alpha}$ for every $\mu$ in $M_{\alpha}^{\prime}$ by Lemma 4, we have

$$
\chi(\mu * \nu)=\chi_{n}(\mu * \nu)=(\mu * \nu) \uparrow(n)=\hat{\mu}(n) \hat{\nu}(n),
$$

and thus $\chi(\mu)=\hat{\mu}(n)$. Clearly, the Gelfand topology is discrete. Therefore (i) is proved. Since $\mathcal{M}\left(M_{\alpha}\right)$ is the one point compactification $\mathcal{M}\left(M_{\alpha}^{\prime}\right) \cup\left\{\chi_{\infty}\right\}$ of $\mathcal{M}\left(M_{\alpha}^{\prime}\right)$, (i) implies (ii). The proof is complete.

5. Idempotent measures and Sidon sets. For $\mu$ in $M_{\alpha}$, if $\mu * \mu=\mu$, it is called an idempotent measure in $M_{\alpha}$. It follows from Lemma 3(ii) that a measure $\mu$ in $M_{\alpha}$ is idempotent if and only if $\hat{\mu}(n)=0$ or 1 for every $n$. The following consideration is motivated by characterizations of the idempotent measures related to ultraspherical polynomials [2] or Jacobi polynomials [6].

Let $\mu$ be an idempotent measure in $M_{\alpha}$ and decompose $\mu$ to $\mu=\nu+\mu(\{0\}) \delta_{0}$, $\nu$ in $M_{\alpha}^{\prime}$. By the convolution equation $\mu * \mu=\mu$, we have

$$
\nu+\mu(\{0\}) \delta_{0}=\nu * \nu+2 \mu(\{0\}) \nu+\mu(\{0\})^{2} \delta_{0} .
$$

Thus $\mu(\{0\})=\mu(\{0\})^{2}$, and so $\mu(\{0\})=0$ or 1 . If $\mu(\{0\})=0$, then $\lim _{n \rightarrow \infty} \hat{\mu}(n)=$ $\lim _{n \rightarrow \infty} \hat{\nu}(n)=0$ by Lemma 5 . Since $\hat{\mu}(n)$ takes only values 0 or 1 , we have $\hat{\mu}(n)=0$ except for a finite number of $n$ 's. Thus we have

$$
d \mu(x)=\frac{1}{\Gamma(\alpha+1)} \sum L_{n}^{\alpha}(x) e^{-x / 2} x^{\alpha} d x
$$

where $\sum$ is a finite sum. If $\mu(\{0\})=1$, then $\hat{\nu}(n)$ takes only values 0 or -1 . Since $\lim _{n \rightarrow \infty} \hat{\nu}(n)=0$, we have

$$
d \mu(x)=-\frac{1}{\Gamma(\alpha+1)} \sum L_{n}^{\alpha}(x) e^{-x / 2} x^{\alpha} d x+d \delta_{0}(x),
$$

where $\sum$ is a finite sum. Therefore we have the following. form

THEOREM 5. Let $\alpha \geq 0$. If $\mu$ is an idempotent measure in $M_{\alpha}$, then $\mu$ has the

$$
d \mu(x)=\frac{1}{\Gamma(\alpha+1)} \sum a_{n} L_{n}^{\alpha}(x) e^{-x / 2} x^{\alpha} d x+a_{\infty} d \delta_{0}(x),
$$

where $a_{n}=1$ or $-1, a_{\infty}=0$ or 1 and $\sum$ is a finite sum.

A subset $E$ of the nonnegative integers will be called a Sidon set with respect to $L_{\alpha}$ if every sequence $\left\{a_{n}\right\}_{n \in E}$ on $E$ vanishing at infinity is the restriction of the Fourier Laguerre coefficients of a function $f$ in $L_{\alpha}$ to $E$; that is, $a_{n}=\hat{f}(n)$ for all $n$ in $E$. The concept of Sidon sets with respect to $L_{\alpha}$ is dual to that of Helson sets with respect to $A^{(\alpha, \tau)}$. We have a theorem which is dual to Theorem 3 (cf. [2 and 10, 5.7]).

THEOREM 6. Let $\alpha \geq 0$ and let $E$ be a subset of the nonnegative integers. Then the following are equiuvalent:

(i) $E$ is a Sidon set with respect to $L_{\alpha}$.

(ii) For every bounded sequence $\left\{a_{n}\right\}_{n \in E}$ on $E$, there is a measure $\mu$ in $M_{\alpha}$ such that $a_{n}=\hat{\mu}(n)$ for all $n$ in $E$.

(iii) $E$ is finite. 
ProOF. By the usual Sidon set argument, we have that (i) implies (ii) (cf. [10, Proof of 5.7.3]). We will show that (ii) implies (iii). Suppose that $E$ is infinite and put $E=\left\{n_{j}\right\}_{j=1}^{\infty}, n_{1}<n_{2}<n_{3}<\ldots$. Define the sequence $\left\{a_{n}\right\}_{n \in E}$ by $a_{n_{j}}=0$ for odd $j$ and $a_{n_{j}}=1$ for even $j$. If there is a measure $\mu$ in $M_{\alpha}$ such that $\hat{\mu}(n)=a_{n}$ for all $n$ in $E$, we have a contradiction since $\lim _{j \rightarrow \infty} \hat{\mu}\left(n_{j}\right)$ exists by Lemma 5 . If $E$ is finite, then

$$
f(x)=\frac{1}{\Gamma(\alpha+1)} \sum_{n \in E} a_{n} L_{n}^{\alpha}(x)
$$

belongs to $L_{\alpha}$, and thus (iii) imples (i). The proof is complete.

\section{REFERENCES}

1. R. Askey and G. Gasper, Convolution structures for Laguerre polynomials, J. Analyse Math. 31 (1977), 48-68.

2. C. F. Dunkl, Operators and harmonic analysis on the sphere, Trans. Amer. Math. Soc. 125 (1966), 250-263.

3. A. Erdélyi, W. Magnus, F. Oberhettinger and F. G. Tricomi, Higher transcendental functions, vol. II, McGraw-Hill, New York, 1953.

4. G. Gasper, Banach algebras for Jacobi series and positivity of a kernel, Ann. of Math. (2) 95 (1972), 261-280.

5. E. Görlich and C. Markett, A convolution structure for Laguerre series, Indag. Math. 44 (1982), 161-171.

6. S. Igari, Certain Banach algebras and Jacobi polynomials, Lecture Note Ser. No. 110, RIMS, Kyoto, 1971, pp. 36-46. (Japanese)

7. S. Igari and Y. Uno, Banach algebra related to the Jacobi polynomials, Tôhoku Math. J. 21 (1969), 668-673.

8. J.-P. Kahane, Séries de Fourier absolument convergentes, Ergeb. Math. Grenzgeb. 50, Springer-Verlag, Berlin, Heidelberg and New York, 1970.

9. B. Muckenhoupt, Mean convergence of Hermite and Laguerre series. I, Trans. Amer. Math. Soc. 147 (1970), 419-431.

10. W. Rudin, Fourier analysis on groups, Interscience, New York, 1962.

11. A. Schwartz, The structure of the algebra of Hankel transforms and the algebra of HankelStieltjes transforms, Canad. J. Math. 23 (1971), 236-246.

12. G. Szegö, Orthogonal polynomials, Amer. Math. Soc. Colloq. Publ., vol. 23, Amer. Math. Soc., Providence, R.I., 1975.

13. G. N. Watson, Another note in Laguerre polynomials, J. London Math. Soc. 14 (1939), 19-22.

Department of Mathematics, College of liberal Arts, KanazaWa UniverSITY, KANAZAWA 920, JAPAN 